Research Article

\title{
Development of Growth Theory for Vapor-Liquid-Solid Nanowires: Wetting Scenario, Front Curvature, Growth Angle, Linear Tension, and Radial Instability
}

\author{
Valery A. Nebol'sin, Nada Swaikat (iD, and Alexander Yu. Vorob'ev \\ Voronezh State Technical University, Department of Radio Engineering and Electronics, Voronezh 394026, Russia \\ Correspondence should be addressed to Nada Swaikat; nada.s84@mail.ru
}

Received 25 October 2019; Accepted 27 February 2020; Published 28 March 2020

Academic Editor: Marco Rossi

Copyright (c) 2020 Valery A. Nebol'sin et al. This is an open access article distributed under the Creative Commons Attribution License, which permits unrestricted use, distribution, and reproduction in any medium, provided the original work is properly cited.

\begin{abstract}
In this paper, we report that under wetting conditions (or modes) of nanowire (NW) growth, when a nonplanar crystallization front emerges under a catalyst droplet, a shift in the three-phase line (TPL) of the vapor-liquid-crystal interface occurs under thermodynamically stable conditions when the angle with respect to the droplet surface, termed the growth angle, is fixed. The growth angle of the NWs is determined not from a geometrical perspective but on the basis of the physical aspects of the processes occurring around the TPL, revealing a size dependence caused by the influence of linear tension of the three-phase contact of a vapor-liquid crystal. The observed radial periodic instability of the NWs is described according to the size dependence of the thermodynamic growth angle, which induces negative feedback in the system. Under the influence of linear tension and positive feedback, the tips or needles of NWs can be formed.
\end{abstract}

\section{Introduction}

The processes of nanowire (NW) growth in semiconducting materials through a vapor-liquid-crystal (VLS) mechanism described in most studies, including [1-11], are based on the established role of the free surface energy of the interfaces and on the flat border of the crystallization front under a catalyst droplet (nonwetting condition (or mode) of whisker growth $[1,2,4,5,9,10])$. In principle, however, this approach is incorrect, because an analysis of the free surface energy can be justified only when one phase is completely dispersed within the volume of the other phase. The growth of NWs is a well-known example of crystallization, in which three phases simultaneously participate in equilibrium in a multicomponent system. The material of each phase near the closed line of the three-phase contact is under the influence of the materials of the two other phases; thus, its state differs from the state within the volume and from the state near the interface of the other two phases [12]. The presence of a three-phase border is the most pronounced feature of NW growth. Hence, the quasi-one-dimensional growth of nanosized crystals cannot be correctly described without considering the free energy, corresponding to a linear tension of the curved interface of the three phases. Here, it is necessary to mention that the effects of the surface curvature of the NWs and the catalyst droplet on the surface energy should appear at radii of the order of unity and one-tenth of a nanometer, which corresponds to the intermolecular distance. In the case of linear tension, the effect of the curvature of the three-phase border along the wetting perimeter of the droplet occurs at radii of tens of nanometers, which corresponds to the radius of action of the surface forces.

During the VLS growth of the NWs, with the small contact angles of the catalyst droplets at the top of the NWs, a three-phase VLS border along the wetting perimeter of the droplet adjoins the singular face of the crystallization front. In such a case, a condition similar to a melt pinned to a crystal edge occurs when the methods developed by Czochralski and Stepanov are used, i.e., when the catalyst droplet 
wets the facet of the crystallization front and does not wet a sidewall surface of the NWs (nonwetting condition (or mode) of NW growth $[10,11,13])$. For a nonwetting growth model, the linear energy that occurs when the catalyst droplet does not wet the sidewall surface of the NWs and a flat crystallization front appears under the droplet was carefully analyzed in $[7,14,15]$. However, it is well known that at large contact angles of catalyst droplets on the top of the NWs at the crystallization front, curved sections and even oblique densely packed faces can appear, leading to the formation of facets on the sidewall of the crystal $[3,16]$. A nonplanar geometry of a crystallization front was realized in the wetting model of NW growth $[10,11]$. Nevertheless, in the literature, the linear energy of a three-phase border under wetting conditions has not been described.

In the present article, we explain that under the wetting conditions for steady NW growth (without considering the kinetic effects), a shift in the three-phase line (TPL) of the interface boundaries occurs at a fixed angle with the surface of the catalyst droplet. Additionally, we reveal the size dependence of the thermodynamic growth angle of the NWs, determined according to the influence of the linear tension of the three-phase contact vapor-liquid crystal. We also show that the growth angle of the NWs should be distinguished from the wetting angle, which characterizes the local equilibrium toward the movement of the liquid droplet over the surface of a solid body and is not directly related to crystallization. Furthermore, we demonstrate that the observed radial periodic instability of the NWs can be explained on the basis of the size dependence of the thermodynamic growth angle, which induces a negative feedback mechanism in the system. Tip or needle NWs can be formed under the influence of linear tension and the action of a positive feedback mechanism.

\section{Wetting Model of NW Growth}

The cross-sectional dimensions of NWs are usually designed according to the linear dependence on the size of the catalyst droplet. As a rule, the ratio of the wire diameter to the droplet diameter is $\sim(0.7: 0.9)[13,17,18]$. We assume that during the growth of cylindrical NWs, the border of the three-phase contact takes the form of a circle. This corresponds to the thermodynamically stable form of a TPL, which is the shortest length for a given area of a crystal-liquid interface.

Assume that a liquid catalyst droplet with a certain volume is placed on top of the NWs with a composition in accordance with the phase diagram of a metal-semiconductor (Figure 1). As the geometrical parameters of the droplet, we choose the base radius $r$ and angles between the tangent and the meniscus of the droplet of the liquid, passing through some point A along the TPL, with horizontal $\theta$ and vertical $\varphi$ (the NWs are grown in the vertical direction). Suppose that in the wetting regime [10] under the droplet, a stepped crystallization front with a thickness of $z$ is formed. Over time, the TPL shifts in the $z$ direction, according to the angle $\delta$ between the vertical and the tangent or the angle $\psi$ between the horizontal and the tangent, to the surface of NWs growing at point A (where $\psi$ is the inclination angle of the curved surface of the crystallization front).

Then, the shift of $l$ from point $A$ to point $A^{\prime}$ is determined as follows:

$$
l=\frac{z}{\cos \delta}
$$

A shift in the TPL on $l$ is accompanied by a reduction of the crystal-liquid interface area by $2 \pi r l \sin \delta$, an increase in the surface crystal area by $2 \pi r l$, a decrease in the liquid surface area by $2 \pi r l \cos (\varphi+\delta)$, and a decrease in the length of the TPL by $2 \pi r l \sin \delta$. Hence, the change in the surface $\left(\Delta F_{S}\right)$ and the linear component of the Helmholtz free energy $\left(\Delta F_{l}\right)$, which is associated with the indicated shift of point $A$, can be expressed as follows:

$$
\begin{aligned}
\Delta F=\Delta F_{s}+\Delta F_{l}= & 2 \pi r l \alpha_{S}-2 \pi r l \alpha_{L} \cos (\varphi+\delta) \\
& -2 \pi r l \alpha_{S L} \sin \delta-2 \pi l \chi \sin \delta
\end{aligned}
$$

where $\alpha_{S}, \alpha_{L}$, and $\alpha_{S L}$ represent the free surface energies of the vapor-crystal, vapor-liquid, and crystal-liquid interfaces, respectively, and $\chi$ represents the tension of a closed line of the three-phase VLS contact.

In equation (2), the first term represents the increment in the free surface energy of the crystal due to an increase in its surface area through a variation in the droplet to height $\mathrm{d} z$, the second term represents a decrease in the free surface energy of the liquid-vapor interface, the third term represents a decrease in the free surface energy of the crystal-liquid interface, and the fourth term represents a decrease in the extra linear energy due to a reduction in the TPL length.

By substituting (1) into (2), then differentiating (2) with respect to angle $\delta$, and equating the derivative to zero $\left(\mathrm{d}(\Delta F) /\left.\mathrm{d} \delta\right|_{\delta=\delta^{*}}=0\right)$, we determine the shift in the TPL, corresponding to the minimum increment in the free surface and linear energies, as follows:

$$
\alpha_{S} \sin \delta^{*}=-\alpha_{L} \sin \varphi+\alpha_{S L}+\frac{\chi}{r}
$$

In equation (3), $\chi / r$ represents the two-dimensional pressure $\alpha_{\chi}$ occurring from the bend of the contact line (analogous to the capillary Laplace pressure arising from the curvature of the interface surface) [12], and $1 / r$ represents the curvature of the line. The absolute value of the linear tension $\chi$ is estimated to be between $1 \times 10^{-11}$ and $1 \times 10^{-9} \mathrm{~J} / \mathrm{m}$ $[7,19]$.

Equation (3) indicates that the angles $\delta^{*}$ and $\varphi$, along with the free surface energies $\alpha_{S}, \alpha_{L}$, and $\alpha_{S L}$ and the linear energy $\alpha_{\chi}$, determine the equilibrium condition of the droplet at each point of the TPL on top of the whisker.

Equation (3) implies an equilibrium condition of the droplet of a liquid lying on a horizontal solid surface (at $\delta^{*}=90^{\circ}$ ).

$$
\alpha_{S}=\alpha_{L} \cos \theta_{0}+\alpha_{S L}+\frac{\chi}{r} .
$$

We obtained modified Young's equation for the mechanical equilibrium of a droplet of liquid on a flat surface, 


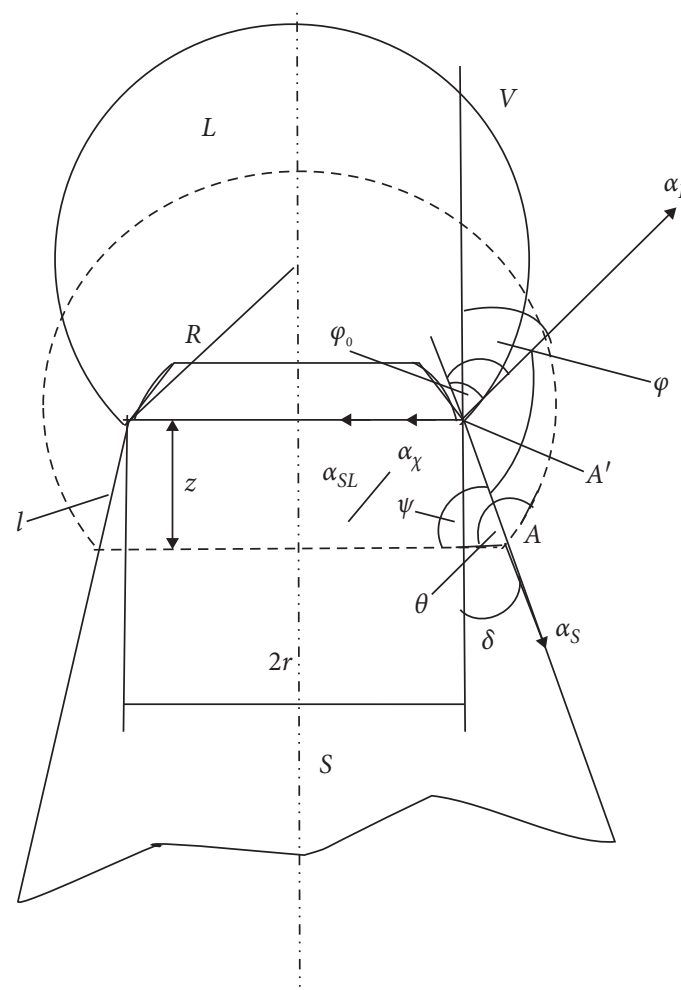

(a)

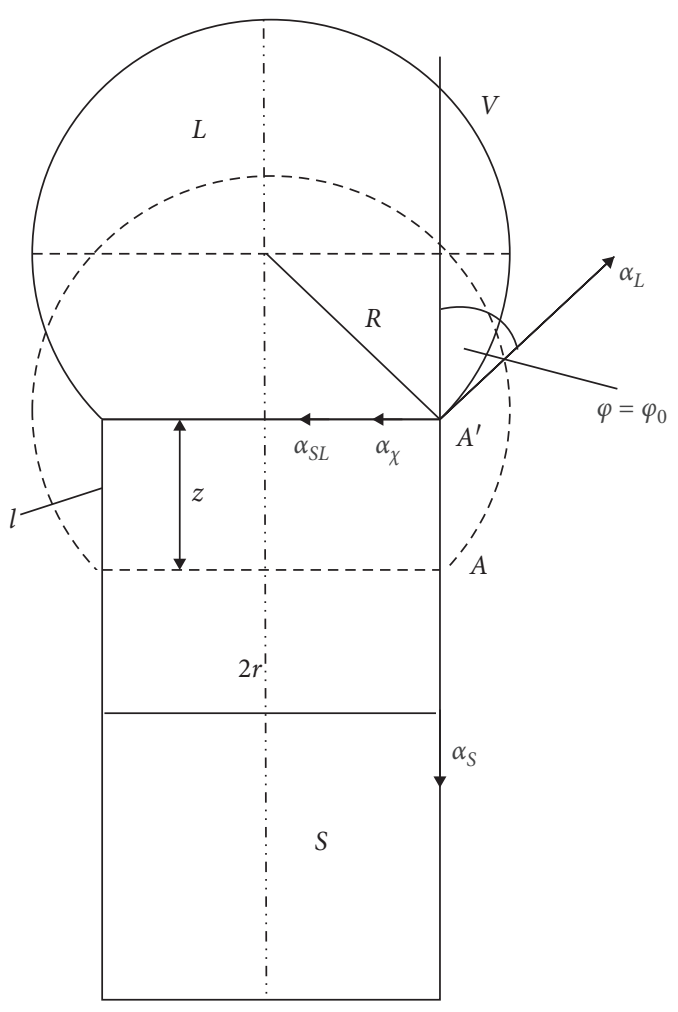

(b)

FIgURE 1: Wetting model of VLS growth of NWs: (a) conical $(\delta>0)$ and (b) constant radius $(\delta=0)$.

taking into account the contribution of the linear tension $\chi$ $[20,21]$. Here, $\theta_{0}$ represents the contact angle (Figure 2(a)).

At $\delta^{*}=0$, a shift in the TPL occurs in the vertical direction (along the axis of the NWs), and the equilibrium of the droplet is thus determined as follows:

$$
\alpha_{L} \sin \varphi=\alpha_{S L}+\frac{\chi}{r},
$$

which coincides with a similar equation obtained for the nonwetting growth of cylindrical NWs [10].

For a system of Au-Si, where $r=1 \times 10^{-7} \mathrm{~m}, \alpha_{L}=0.91 \mathrm{~J} / \mathrm{m}^{2}$, $\alpha_{S L}=0.45 \mathrm{~J} / \mathrm{m}^{2}$, and two angles $\varphi$ are $32^{\circ}$ and $35^{\circ}$ [22], using equation (5), we calculate the linear tension $\chi$ and linear energy $\alpha_{\chi}$. For $\varphi=32^{\circ}$, we obtain $\chi=3.22 \times 10^{-9} \mathrm{~J} / \mathrm{m}$ and $\alpha_{\chi}=3.22 \times 10^{-11} \mathrm{~J} / \mathrm{m}^{2}$. Thus, $\alpha_{\chi}$ is approximately $7 \%$ of the free interfacial energy of the crystal-liquid interface. For $\varphi=35^{\circ}$, we obtain $\chi=7.20 \times 10^{-9} \mathrm{~J} / \mathrm{m}$. Accordingly, $\alpha_{\chi}=7.20 \times 10^{-11} \mathrm{~J} / \mathrm{m}^{2}$, which is $\sim 16 \%$ of the set value of the free surface energy $\alpha_{\mathrm{SL}}$.

\section{Growth Angle of NWs}

When $0 \leq \delta^{*} \leq 90^{\circ}$, the configuration of the small catalyst droplet on top of the NWs must strictly correspond to the angles $\theta$ and $\psi$. The difference between them $\left(\theta-\psi=\varphi+\delta^{*}\right)$ is the thermodynamic growth angle of the NWs $\varphi_{0}$, that is, the angle between the tangent drawn to the meniscus of the liquid phase at the selected point at the interface of the three phases and the direction of the equilibrium shift of the TPL
$[23,24]$. The increment in the free energy of the three-phase system at the shift of the TPL under $\varphi_{0}$ is zero; further, the formation of the NW surface under other angles differing from $\varphi_{0}$ leads to an increase in the free energy during this process and therefore cannot occur. Hence, $\varphi_{0}$ is the equilibrium angle of the TPL shift during the growth of the NWs. The tendency of the NWs to grow with a constant diameter is determined by the fact that during the growth of the cylindrical crystals, the increment in the areas of the interfaces is minimal.

The phase of zincblende GaAs NWs is formed at small $\left(<100^{\circ}\right)$ and large $\left(>125^{\circ}\right)$ contact angles $\theta$ and, accordingly, at $\varphi_{0}<10^{\circ}$ and $\varphi_{0}>125^{\circ}$, whereas the pure wurtzite phase is observed for intermediate contact angles [25].

To obtain an equation for the growth angle of the NWs $\varphi_{0}$ in an explicit form, we first apply the axial and radial components of the projections of the force vectors, corresponding to the free surface energies of interfaces $\alpha_{S}$ and $\alpha_{S L}$ at point $\mathrm{A}$ on the TPL for large angles of macroscopic droplets $\varphi_{0} \longrightarrow \varphi_{0}^{\infty}$ (without taking into account the contributions of the linear tension and the anisotropy of the free surface energy at the interfaces). This yields the following system of equations:

$$
\left\{\begin{array}{l}
-\alpha_{S}+\alpha_{L} \cos \varphi_{0}^{\infty}+\alpha_{S L} \sin \psi=0, \\
-\alpha_{L} \sin \varphi_{0}^{\infty}+\alpha_{S L} \cos \psi=0,
\end{array}\right.
$$

where $\varphi_{0}^{\infty}$ represents the angle between the tangent drawn to the meniscus of the liquid phase at the selected point $A$ at the 


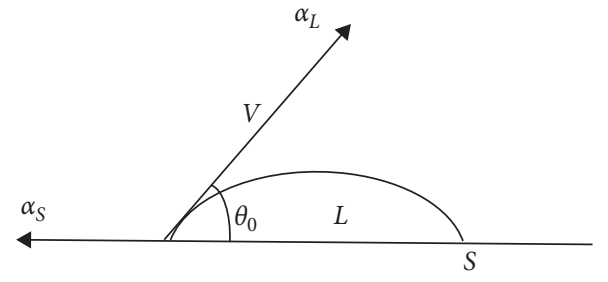

(a)

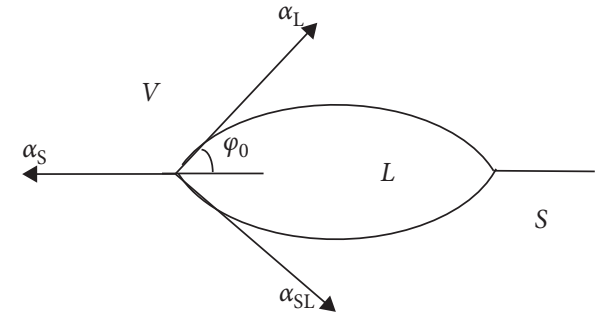

(b)

FIGURE 2: Scheme of the three phases in the conjugation: solid, liquid, and vapor: (a) at a thermodynamic position of nonequilibrium but with mechanical equilibrium of the droplet on the crystal surface and (b) at a position of thermodynamic equilibrium of the catalyst droplet on the crystal surface.

interface of the three phases and the direction of the equilibrium shift of the TPL without the influence of the linear tension.

Using the trigonometric identity $\cos ^{2} \varphi_{0}^{\infty}+\sin ^{2} \varphi_{0}^{\infty}=1$ and excluding the angle $\psi$ from equation (6), we express the growth angle $\varphi_{0}^{\infty}$ of the NWs in an explicit manner as follows:

$$
\varphi_{0}^{\infty}=\arccos \left(\frac{\alpha_{S}^{2}+\alpha_{L}^{2}-\alpha_{S L}^{2}}{2 \alpha_{S} \alpha_{L}}\right)
$$

According to (7), the angle $\varphi_{0}^{\infty}$ is a constant value for the present crystallized substance under the given thermodynamic conditions of steady growth of NWs; this is determined not from a geometric viewpoint but rather according to the physical nature of the processes occurring within the neighborhood of the TPL. The growth angle of the whiskers $\varphi_{0}^{\infty}$ should be distinguished from the wetting angle $\theta_{0}$, which characterizes the local equilibrium toward the movement of a liquid droplet over the surface of the solid body, and is not directly related to crystallization. An exception is the case of a catalyst droplet position with thermodynamic equilibrium on the crystal surface (Figure 2(b)).

It follows from (7) that if $\alpha_{S}>\alpha_{L}$ and $\alpha_{L}>\alpha_{S L}$, to ensure the circularity of the force vectors corresponding to the free surface energies, the equilibrium under the droplet must be convex toward the droplet. In contrast, if $\alpha_{S}<\alpha_{L}$, the front must be concave. If the curved section of the front crystallization below the droplet is concave (the curvature center is outside the crystal), there is a high saturation above it (Kelvin's effect). Thus, this section transforms into the single face of the crystallization front (Figure 1(a)).

The angle $\psi$ is expressed by the second part of equation (6). Instead of the growth angle $\varphi_{0}^{\infty}$, we substitute its value from (7). Hence, we obtain the following:

$$
\psi=\arccos \left[\frac{\alpha_{L}}{\alpha_{S L}} \sqrt{1-\left(\frac{\alpha_{S}^{2}+\alpha_{L}^{2}-\alpha_{S L}^{2}}{2 \alpha_{S} \alpha_{L}}\right)^{2}}\right] .
$$

From equation (8), it is clear that with an isotropic approximation, the inclination angle $(\psi)$ of the curved crystallization front of the NWs near the TPL is determined by the equilibrium conditions at the interface border.
According to equation (8), at $\varphi_{0}^{\infty}=0$, the inclination angle is $\psi=90^{\circ}$. Hence, the droplet in this case represents a hemisphere on top of the NWs, and the radius of the curvature of the TPL is maximized [10]. Growth of the NWs under these conditions is impossible, because, with an increase in the wetting perimeter of the growth substrate, the interface surface energy continuously decreases (full wetting). The radius of the line of the three-phase contact of the droplet on the substrate tends toward infinity. In this case, the liquid-phase catalyst spreads over the surface of the substrate, and crystallization can lead to epitaxial growth of the film when a solution of an intermediate metallic substance is applied to the substrate as a thin layer [26].

If $\varphi_{0}^{\infty}=90^{\circ}$, the contact angle $\varphi$ is unstable, and any contact between the droplet and crystal leads to an increase in the interfacial energy (full nonwetting). The radius of curvature of the three-phase contact line is zero $(r=0)$, and, according to (8), $\psi=\arccos \left(\alpha_{L} / \alpha_{S L}\right)$. Because $\alpha_{L}>\alpha_{S}$, the angle $\psi$ is not defined, and stable growth of the NWs is impossible. At $\varphi_{0}^{\infty}=90^{\circ}$ a phase transition is only possible at the border with the vapor $[10,25]$.

At $\psi=0$, equation (8) moves toward a well-known condition of catalyst droplet equilibrium $\varphi_{0}^{\infty}=\arcsin \left(\alpha_{S L} / \alpha_{L}\right)$, which characterizes the nonwetting model for the stable growth of whiskers when a flat face of the crystallization front adjoins the TPL $[10,26]$.

Thus, an analysis of the thermodynamic angle of crystallization reveals that the growth of the NWs is thermodynamically possible if the contact angle $\varphi$ is within the interval $0<\varphi<90^{\circ}\left(90^{\circ}<\theta<180^{\circ}\right)$. At $\varphi \geq 90^{\circ}$, the contact angle of the droplet with the crystal is unstable, and any contact between the droplet and crystal leads to an increase in the free surface energy; hence, such contact is impossible. At $\varphi \leq 0$, no stable contact angle of the catalyst droplet occurs on top of the NWs, and, in this case, full wetting is realized $[10,26]$.

Table 1 presents the calculation results for the growth angle $\varphi_{0}^{\infty}$, the inclination angle of the curved surface of the crystallization front $\psi$ based on equation (8), and the contact angle $\theta_{0}$ based on equation (4) at $\chi / r=0$ for certain parameters of the growth of cylindrical $\mathrm{Si}, \mathrm{GaAs}$, and InAs NWs with the participation of $\mathrm{Au}$ and $\mathrm{Ga}$ particles, the calculation of angle $\theta$, and the following free energy values: 
TABLE 1: Inclination angle of the curved surface of the crystallization front, growth angle of Si, GaAs, and InAs NWs in the presence of Au and Ga particles, and contact angle of the catalyst droplet on the flat surface.

\begin{tabular}{lccccc}
\hline NW type & Cubic Si (CUB) & $\begin{array}{c}\text { Cubic } \\
\text { GaAs } \\
(\mathrm{ZB})\end{array}$ & Hexagonal GaAs (WZ) & Cubic InAs (ZB) & Hexagonal InAs (WZ) \\
\hline Type of particles & $\mathrm{Au}$ & $\mathrm{Au} \mathrm{Ga}$ & $\mathrm{Au}$ & $\mathrm{Au}$ & $\mathrm{Ga}$ \\
Growth angle $\varphi_{0}^{\infty}$, in degrees & 19 & $28 \quad 20$ & 57 & 52 & 49 \\
Angle $\psi$, in degrees & 49 & $61 \quad 72$ & 39 & 64 & 19 \\
Contact angle of droplet $\theta_{0}$, in degrees & 34 & $46 \quad 37$ & 69 & 84 \\
\hline
\end{tabular}

$\alpha_{L}^{\mathrm{Au}}=0.910 \mathrm{~J} / \mathrm{m}^{2}, \alpha_{S}=1.2 \mathrm{~J} / \mathrm{m}^{2}$, and $\alpha_{S L}=0.45 \mathrm{~J} / \mathrm{m}^{2}[2,24]$ for $\mathrm{Si} ; \alpha_{L}^{\mathrm{Au}}=0.910 \mathrm{~J} / \mathrm{m}^{2}, \alpha_{L}^{\mathrm{Ga}}=0.800 \mathrm{~J} / \mathrm{m}^{2}, \alpha_{S L}^{\mathrm{Au}}(\mathrm{ZB})=0.866 \mathrm{~J} / \mathrm{m}^{2}$, $\alpha_{S L}^{\mathrm{Au}}(\mathrm{WZ})=0.980 \mathrm{~J} / \mathrm{m}^{2}, \quad \alpha_{S L}^{\mathrm{Ga}}(\mathrm{ZB})=0.780 \mathrm{~J} / \mathrm{m}^{2}, \quad \alpha_{S}(\mathrm{ZB})=$ $1.500 \mathrm{~J} / \mathrm{m}^{2}$, and $\alpha_{S}(\mathrm{WZ})=1.300 \mathrm{~J} / \mathrm{m}^{2}$ [3] for GaAs; and $\alpha_{S}(\mathrm{ZB})=1.190 \mathrm{~J} / \mathrm{m}^{2}, \alpha_{S}(\mathrm{WZ})=0.910 \mathrm{~J} / \mathrm{m}^{2}, \alpha_{L}^{\mathrm{Au}}=1.000 \mathrm{~J} / \mathrm{m}^{2}$, and $\alpha_{S L}^{\mathrm{Ga}}(\mathrm{ZB})=0.800 \mathrm{~J} / \mathrm{m}^{2}$ for InAs [3].

In our model, the angle $\varphi_{0}^{\infty}$ determines the direction of the TPL offset and represents the NW growth angle $\varphi_{0}^{\infty}$ (Figure 1(b)) under the condition of a constant growth angle. When the volume of the catalyst droplet is maintained, the NWs grow in a cylindrical shape with an isotropic side surface. However, at certain angles $\psi$ on the curved crystallization front under a droplet in the TPL neighborhood, an internal inclined (oblique) tight-packed $\mathrm{AA}^{\prime}$ face may occur, characterized by small Miller indices $\{h k l\}$; thus, the sidewall of the NWs will be inclined at a certain angle to this face [27].

The appearance of such an $\mathrm{AA}^{\prime}$ face is possible owing to the dependence of the specific free surface energy on the orientation of the face, as it is known that the singular faces correspond to a sharp minimum, and the angular derivative $\partial \alpha / \partial \psi$ reaches the greatest value (Figure 3) [28].

So that a peripheral low-index face emerging into the TPL could appear at a point on the curved area of the crystallization front under the catalyst droplet on the top of NWs, it is necessary that the front sections under the droplet near this point do not serve as a source of steps for the face. This requires the initial curved crystallization front of NWs and the TPL itself to be convex (the curvature center of the surface is situated in the crystal). If the formed singular face $\{h k l\}$ comes out on the TPL, then the latter changes its round shape and straight sections should appear on it $[8,27,29,30]$.

It is necessary that the initial curved crystallization front of the NWs and the TPL is convex (the center of curvature of the surface is in the crystal). If the single face $\{h k l\}$ reaches the TPL, the TPL changes from its round shape and adopts straight sections that should appear.

The outside surface of the NWs is formed as a result of the TPL movement. Hence, under the appropriate conditions, the emergence of oblique singular faces on the side surface of the growing NWs is energetically advantageous (e.g., faces (111) A and (111) B for crystals GaAs of the zincblende (cubic) crystal structure and the $<111>$ orientation of the growth axis $[8,30])$. The exit of the inclined facets $\{111\}$ on the sidewall surface in the untwined Si, Ge, and GaAs NWs leads to facet formation with the crystallographic orientation $\{1 \overline{12}\}$ [27]. The structure of the $\{1 \overline{12}\}$

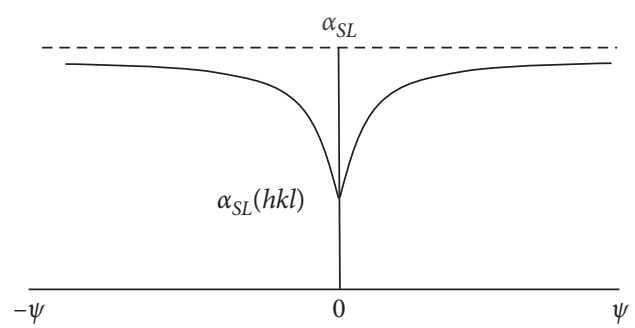

FIGURE 3: Anisotropy of crystal's free surface energy nearby the singular faces with small crystallographic indexes $\{h k l\}$.

facets is an interchange of (111) faces for Si and Ge, and (111) $\mathrm{A}$ is an interchange for GaAs, which is bent at an angle of $\sim 19.5^{\circ}$ with respect to the growth axis of the NWs and has "correcting" steps between them. The formation of the side faceting of NWs is described in $[8,16,25,27,29,30]$.

A necessary condition for the appearance of an oblique tight-packed face $(h k l)$ on the sidewall surface of the NWs is that the equilibrium wetting angle of the oblique facet according to Young's modulus should increase the actual wetting angle of the facet $\theta-\psi$.

During movement of the catalyst droplet along the inclined surface $(h k l)$ its radius and wetting perimeter decrease, while angle between the tangent to droplet surface and face $\theta-\psi$ in condition of volume constancy of liquid phase increases consecutively until it reaches value $\theta(h k l)$ of Young's in point $A^{\prime}$. When condition $\theta=\theta(h k l)$ is reached at some point $A^{\prime}$, the further droplet movement along the face $(h k l)$ is difficult due to increase of free surface energy in this process after which the droplet flows onto the face. Then, the crystallization starts on the oblique face and the TPL moves at angle $\theta-\varphi_{0}^{\prime}$, forming an expanding area of isotropic surface of NWs. Growth angle $\varphi_{0}^{\prime}$ is less than the initial angle $\varphi_{0}^{\infty}$, as supersaturation on face $(h k l)$ increases.

The condition for the constancy of the growth angle $\varphi_{0}^{\infty}$, in this case, is satisfied only at the stage of growth of the transition section ("correcting" steps) between the two sections of the crystal narrowing along the face. The facet NWs can be controlled by changing the wetting angle $\theta^{\prime}$ of the catalyst droplet at the top of the crystal.

\section{Line Tension TPL}

For the wetting model of NW growth, the full equilibrium conditions of a closed line of the three-phase contact VLS at point $A$ under a droplet can be obtained by introducing the 
linear energy $\chi / r$ into the second equation of the system of equations in equation (6) [10]:

$$
\left\{\begin{array}{l}
-\alpha_{S}+\alpha_{L} \cos \varphi_{0}+\alpha_{S L} \sin \psi=0 \\
-\alpha_{L} \sin \varphi_{0}+\alpha_{S L} \cos \psi+\frac{\chi}{r}=0 .
\end{array}\right.
$$

At an angle $\varphi_{0}$ in the system of equation (9), the true growth angle $\varphi_{0}$ of the NWs differs from the angle $\varphi_{0}^{\infty}$ in equation (6) for the case of a macroscopic crystal and droplet or a direct contact line, i.e., for $r \longrightarrow \infty$.

By excluding the angle $\psi$ from the system of equation (9) and considering equation (7), we express $\chi / r$ as follows:

$$
\frac{\chi}{r}=\alpha_{L}\left(\sin \varphi_{0}-\sqrt{\sin ^{2} \varphi_{0}-\frac{2 \alpha_{S}\left(\cos \varphi_{0}^{\infty}-\cos \varphi_{0}\right)}{\alpha_{L}}}\right) .
$$

Equation (10) provides the implicit dimensional dependence of the growth angle of NWs, which is affected by the linear tension during the heterogeneous formation process of a new phase. According to equation (10), at $\varphi_{0} \longrightarrow \varphi_{0}^{\infty}$ and a constant $\alpha_{L}$, the linear energy tends toward zero $(\chi \longrightarrow 0)$. Additionally, at the positive curvature of the TPL (where the center of the curvature is inside the droplet or crystal), the linear tension $\chi$ can be positive or negative. The value of $\chi$ is positive; i.e., its force contribution $\alpha_{\chi}$ at point $A$ on the TPL is directed radially inward when $\varphi_{0}^{\infty}<\varphi_{0}$. A positive linear tension $(\chi>0)$ leads to a contraction of the circular wetting perimeter of the droplet under a decrease in radius and an increase in angle $\varphi_{0}$ compared with its macroscopic value $\varphi_{0}^{\infty}$. Otherwise, the linear tension is negative $(\chi<0$ and the force vector at point $A$ on the TPL is directed radially outward), and, with a decrease in $r$, a decrease in $\varphi_{0}$ occurs compared with $\varphi_{0}^{\infty}$, which increases the circular wetting perimeter.

If the linear tension is positive, a slight random change in the NW diameter, for example, a decrease, leads to an increase in the $\chi / r$ value; hence, a disbalance and decrease in the droplet wetting perimeter occur. Then, a reduction of the $\mathrm{NW}$ diameter is needed to restore the balance of the forces, which leads to internally contracting growth and the formation of conical and needle crystals, resulting in the droplet jumping off the top of the NW (Figure 4). Conversely, a random increase in the NW diameter leads to a decrease in the $\chi / r$ value and further widening of the NW. The described mechanism of positive feedback causes unstable growth of the NWs.

If $\chi<0$, during NW growth, a mechanism of negative feedback is realized, which stabilizes the crystal growth [7]. In this case, a slight disturbance reducing the radius of the NWs will reduce $\chi / r$ and lead to the recovery of the crosssectional area of the crystal to the initial undisturbed state. Hence, the linear tension $\chi$ should be negative for stable NW growth.

Using equation (10), we plot the dependence of the growth angle $\varphi_{0}$ on $\chi / r$ (Figure 5). To construct the graph in Figure 5 the following values of the free surface energies were used: $\alpha_{L}=0.91 \mathrm{~J} / \mathrm{m}^{2}$ and $\alpha_{S}=1.20 \mathrm{~J} / \mathrm{m}^{2}$, which are typical for $\mathrm{Au}-\mathrm{Si}$ systems $[2,26]$.

As shown in Figure 5, the true growth angle $\varphi_{0}$ of the whisker increased in direct proportion to the dimensionless curvature $\chi^{*}$. However, depending on the linear tension, the thermodynamic growth angle $\varphi_{0}$ could both increase (at $\chi>0$ ) and decrease (at $\chi<0$ ).

If we exclude the angle $\psi$ from the system of equations (equation (9)and consider equation (7), we can express the thermodynamic growth angle of the NWs $\varphi_{0}$ for a small catalyst droplet as follows:

$$
\varphi_{0}=\arccos \left[\cos \varphi_{0}^{\infty}+\frac{(\chi / r)^{2}-2(\chi / r) \alpha_{L} \sin \varphi_{0}}{2 \alpha_{S} \alpha_{L}}\right] .
$$

In deriving (11) on the basis of a detailed analysis of the radicals in a quadratic equation, a negative sign was chosen because a positive sign yields nonphysical results. It follows from (11) that at a positive curvature of the TPL and a decrease in the NW radius, the growth angle $\varphi_{0}$ can both increase and decrease in relation to the angle $\varphi_{0}^{\infty}$.

If $\chi>0$ and $(\chi / r)>2 \sin \varphi_{0}$, i.e., the force contribution of the linear tension in the droplet equilibrium is greater than the double vector projection, corresponding to the free surface energy of the liquid-vapor interface on the plane of the droplet contour, with a decrease in the crystal radius, $\varphi_{0}$ decreases (the second term of the function argument of the arc cos on the right-hand side of (11) with a “+” sign). Conversely, at $\chi>0$ and $\chi / r<2 \sin \varphi$, the growth angle $\varphi_{0}$ increases with a decrease in $r$ (before the "-" sign appears in the second term in (11)). For $\chi<0$, as determined by the influence of the linear tension on the value, the growth angle develops in the opposite direction.

For example, for a dimensionless curvature of the TPL $\chi^{*}=0.2$ and the aforementioned values $\alpha_{L}^{\mathrm{Au}}$ and $\alpha_{S}$ for Si at $\varphi_{0}^{\infty}=10^{\circ}$, the growth angle satisfying equation (11) is $\varphi_{0} 7^{\circ}$, which is smaller than the given value of $\varphi_{0}^{\infty}$. Hence, in the considered example, at a linear tension of $\chi<0$, the TPL is stretched, and the NWs should widen during growth. For the aforementioned conditions but at $\chi^{*}=0.4$, we obtain $\varphi_{0} 17^{\circ}$, which is larger than $\varphi_{0}^{\infty}=10^{\circ}$. Hence, it follows that $\chi>0$, and the NWs narrow during their growth.

If the linear tension on the line of the interfacial contact is negative $(\chi<0)$, the equilibrium of the two-dimensional phases, for example, those having free surface energies $\alpha_{S L}$ and $\alpha_{L} \sin \varphi$ and that divided by a circular contour of the catalyst droplet with a curvature radius $r$, is stable. This conclusion follows from the consideration of the extremum of the Helmholtz free energy with a change in the radius of the contour of the catalyst droplet on top of the NWs in a two-dimensional system (in the action plane) for $\mathrm{d} r$ :

$$
\mathrm{d} F=\left[2 \pi r\left(\alpha_{S L}-\alpha_{L} \sin \varphi\right)+2 \pi \chi\right] \mathrm{d} r=0
$$

From equation (12), we obtain the following:

$$
\alpha_{L} \sin \varphi-\alpha_{S L}=\frac{\chi}{r}
$$

which is analogous to (5) at $\delta^{*}=0$. Then, $\partial^{2} F / \partial r^{2}=$ $-(2 \pi \chi / r)>0$, as $\chi<0$. 


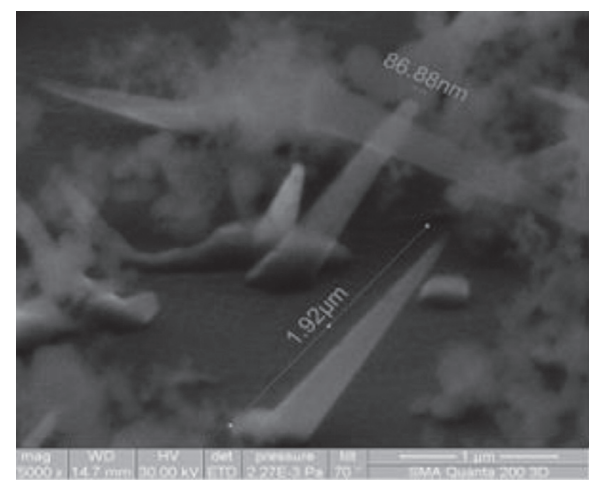

(a)

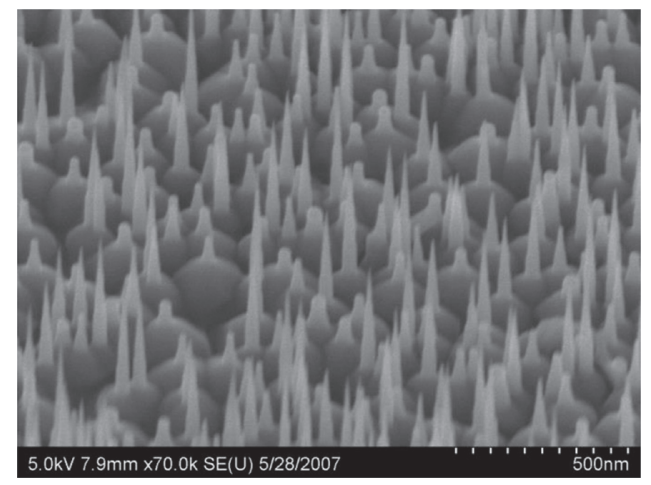

(b)

Figure 4: SEM image of needle NWs: (a) Si and (b) GaAs.

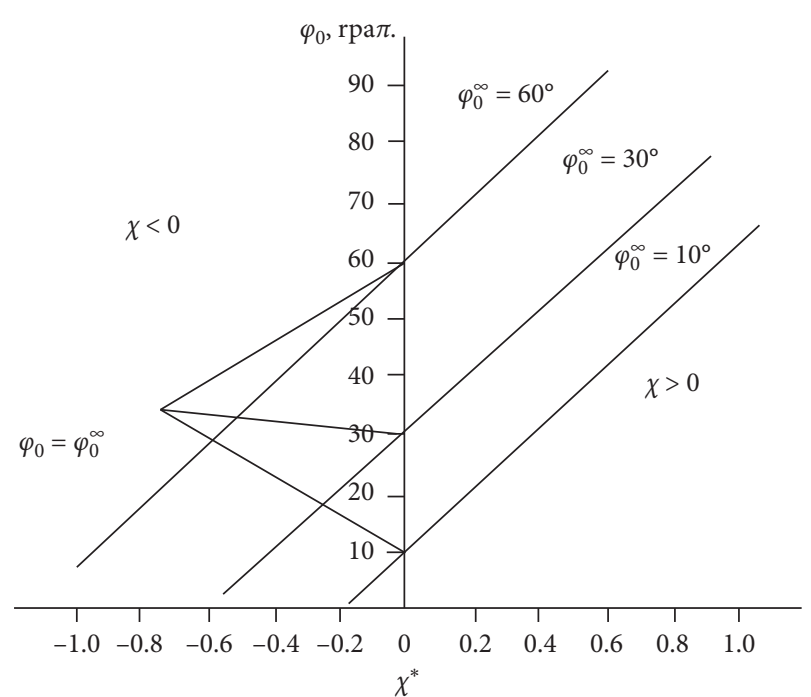

FIgURE 5: Calculated dependences of the thermodynamic growth angle of Si NWs on the dimensionless curvature of the TPL $\chi^{*}=$ $\chi / \alpha_{L} r$ for different values of the whisker growth angle $\varphi_{0}^{\infty}$, which characterizes the macroscopic droplet $(r \longrightarrow \infty)$.

A consideration of the linear tension does not affect the conclusion regarding the stability of NW growth if $\chi>0$, as in the case of $\partial^{2} F / \partial r^{2}<0[31]$.

\section{Model Periodic Instability of NW Growth}

The size dependence of the growth angle can explain the emergence of negative feedback in the system, which leads to the radial periodic instability of Si NWs (Figure 6) [32, 33]. Radial periodic instability is the result of the formation of rhythmically repeated spikes and cavities on the sidewall of the NWs. Periodic instability occurs only for the thinnest NWs (average diameter of $50-100 \mathrm{~nm}$ ). Radial instability was discovered by Givargizov in Si NWs grown using Au particles [32]. These crystals consisted of a series of bundles, and overstretching with approximately equal intervals occurred between them. The change in the crystal diameter was determined by the instability of the wetting contour of the droplet during growth. Although the radius of the contour changed periodically, the bundles differed slightly from each other with regard to diameter and length. Thus, the periodicity had a nonstrict nature, and the sequences of bundles of different diameters were not identical for different crystals. Each crystal had its own law of oscillation. Additionally, small amounts of $\mathrm{AsCl}_{3}$ vapor reduced the contact angle of the droplet and eliminated the instability.

To describe the periodic instability of NW growth, we analyze the initial segment of a narrowing crystal. At the narrowing section toward the thick NWs (where the force contribution $\chi / r$ to the equilibrium at a point on the TPL can still be small), the doubled projection of vector $\alpha_{L}$ on the plane of the droplet contour $\left(2 \alpha_{L} \sin \varphi_{0}\right)$ exceeds the force vector $\alpha_{\chi}$ aligned with the linear tension $\chi$. According to equation (11), the growth angle $\varphi_{0}$ increases with a decrease in $r$. An increase in $\varphi_{0}$ leads to further narrowing of the crystal, a decrease in the wetting perimeter of the droplet, and an increase in the vector projection, corresponding to the free surface energy $\alpha_{L}$ (increase of $2 \alpha_{L} \sin \varphi_{0}$ in equation (11)). However, as the wetting perimeter decreases (and hence the NW diameter decreases), the force contribution of the linear tension $\alpha_{\chi}$ grows faster than the projection of the vector $\alpha_{L}$ onto the plane of the droplet contour. This results in a decrease in the growth angle. The decrease in $\varphi_{0}$ contributes to a shift of the TPL in the direction of the crystal extension. Therefore, when a certain minimum diameter (diameter of overstretching) is reached, the NWs again extend until reaching a maximum diameter (diameter of the bundle). The dynamic equilibrium becomes unstable, and, after stopping, the process repeats.

\section{Conclusions}

It was shown that in the wetting growth regime of NWs, a shift in the TPL occurs at a fixed angle with respect to the surface of the catalyst droplet (angle of growth $\varphi_{0}$ ). Additionally, the direction of the shift in the TPL is determined by the zero increment of the free energy of the system in the aforementioned process, because it is the direction of the equilibrium shift. 


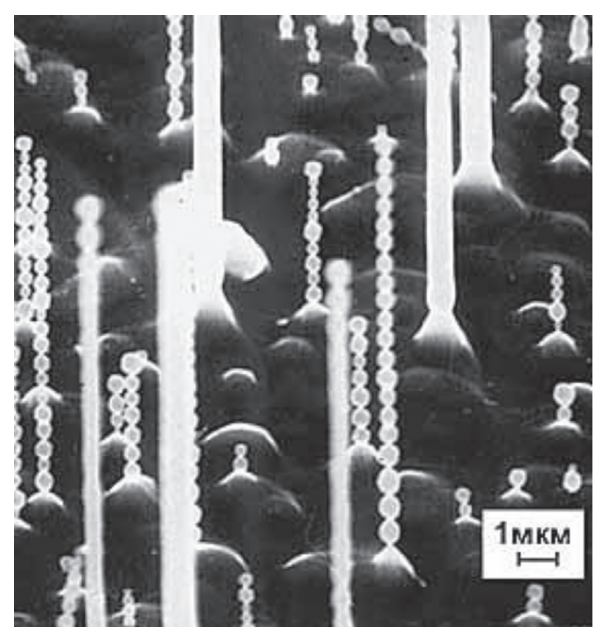

(a)

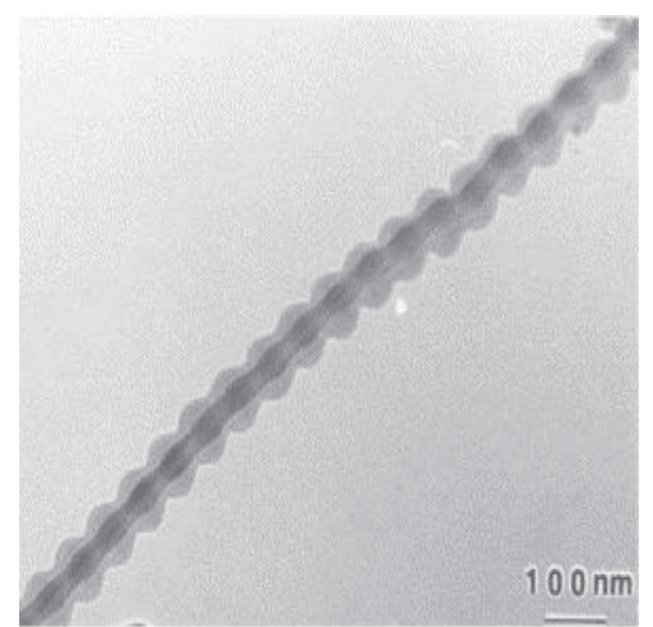

(b)

Figure 6: Radial periodic instability of Si NWs: (a) [32] and (b) [34].

The dimensional dependence of the thermodynamic angle of NW growth is determined by the influence of the linear tension on the process of the heterogeneous formation of a small crystalline phase. It was shown that at a positive curvature of the TPL, with a decrease in the radius of the NWs, the growth angle can either increase or decrease in relation to the thermodynamic growth angle for a macroscopic catalyst droplet or a straight TPL.

It was shown that the observed radial periodic instability of Si NWs can be explained according to the dimensional dependence of the thermodynamic growth angle, which causes a negative feedback mechanism in the system. Tip or needle NWs can be formed under the influence of linear tension and the action of a positive feedback mechanism.

\section{Data Availability}

Any data and information used to support the findings of this study will be provided by the corresponding author upon request.

\section{Conflicts of Interest}

The authors declare that there are no conflicts of interest regarding the publication of this paper.

\section{Acknowledgments}

The study was conducted with financial support from the Russian Foundation for Basic Research (RFBR) as part of the scientific project No. 19-33-90219, "Nanoelectronics and Nanotechnology Devices," at the collective center for use of scientific equipment of Voronezh State Technical University. The authors would like to thank Editage (http://www. editage.com) for English language editing.

\section{References}

[1] V. Schmidt, J. V. Wittemann, and U. Gösele, "Growth, thermodynamics, and electrical properties of silicon nanowires," Chemical Reviews, vol. 110, no. 1, pp. 361-388, 2010.

[2] E. I. Givargizov, "Fundamental aspects of VLS growth," Journal of Crystal Growth, vol. 31, pp. 20-30, 1975.

[3] V. G. Dubrovskii, Nucleation Theory and Growth of Nanostructures, Springer-Verlag, Berlin, Germany, 2014.

[4] J. Johansson, L. S. Karlsson, C. P. T. Svensson et al., "Structural properties of $\langle 111\rangle$ B-oriented III-V nanowires,” Nature Materials, vol. 5, no. 7, pp. 574-580, 2006.

[5] V. A. Nebol'sin, J. Johansson, D. B. Suyatin, and B. A. Spiridonov, "Thermodynamics of oxidation and reduction during the growth of metal catalyzed silicon nanowires," Journal of Crystal Growth, vol. 505, pp. 52-58, 2019.

[6] V. A. Nebol'sin and A. A. Shchetinin, "A mechanism of quasione-dimensional vapor phase growth of $\mathrm{Si}$ and GaP whiskers," Inorganic Materials, vol. 44, no. 10, pp. 1033-1040, 2008.

[7] V. Schmidt, S. Senz, and U. Gösele, "The shape of epitaxially grown silicon nanowires and the influence of line tension," Applied Physics A, vol. 80, no. 3, pp. 445-450, 2005.

[8] D. Jacobsson, F. Panciera, J. Tersoff et al., "Interface dynamics and crystal phase switching in GaAs nanowires," Nature, vol. 531, no. 7594, pp. 317-322, 2016.

[9] B. A. Wacaser, K. A. Dick, J. Johansson, M. T. Borgström, K. Deppert, and L. Samuelson, "Preferential interface nucleation: an expansion of the VLS growth mechanism for nanowires," Advanced Materials, vol. 21, no. 2, pp. 153-165, 2009.

[10] V. A. Nebol'sin, A. I. Dunaev, A. F. Tatarenkov, and S. S. Shmakova, "Scenarios of stable vapour-liquid dropletsolid nanowire growth," Journal of Crystal Growth, vol. 450, pp. 207-214, 2016.

[11] V. G. Dubrovskii, G. E. Cirlin, N. V. Sibirev, F. Jabeen, J. C. Harmand, and P. Werner, "New mode of vapor-liquid-solid nanowire growth," Nano Letters, vol. 11, no. 3, pp. 1247-1253, 2011.

[12] A. Scheludko, V. Chakarov, and B. Toshev, "Water condensation on hexadecane and linear tension," Journal of Colloid and Interface Science, vol. 82, no. 1, pp. 83-92, 1981. 
[13] V. A. Nebol'sin, D. B. Suyatin, A. I. Dunaev, and A. F. Tatarenkov, "Capillary stability of VLS crystallization processes and its comparison to Czochralski and Stepanov growth methods," Journal of Crystal Growth, vol. 463, pp. 46-53, 2017.

[14] V. A. Nebol'sin, A. I. Dunaev, and M. A. Zavalishin, "Effect of the line tension at the vapor-liquid-solid boundary on the growth of silicon nanocrystals," Inorganic Materials, vol. 44, no. 6, pp. 559-562, 2008.

[15] V. A. Nebol'sin, D. B. Suyatin, A. I. Dunaev, S. S. Shmakova, M. A. Zavalishin, and E. V. Ivannikova, "Contribution of the free energy of the three phase line of contact to the thermodynamic equilibrium conditions of a metal solvent droplet in Si and Ge whisker growth," Inorganic Materials, vol. 51, no. 3, pp. 191-196, 2015.

[16] V. A. Nebol'sin, A. A. Shchetinin, A. N. Korneeva et al., "Development of lateral faces during vapor-liquid-solid growth of silicon whiskers," Inorganic Materials, vol. 42, no. 4, pp. 339-345, 2006.

[17] S. Kodambaka, J. Tersoff, M. C. Reuter, and F. M. Ross, "Diameter-independent kinetics in the vapor-liquid-solid growth of Si nanowires," Physical Review Letters, vol. 96, no. 9, p. 096105, 2006.

[18] S. Kodambaka, J. Tersoff, M. C. Reuter, and F. M. Ross, "Germanium nanowire growth below the eutectic temperature," Science, vol. 316, no. 5825, pp. 729-732, 2007.

[19] J. S. Rowlinson and B. Widom, Molecular Theory of Capillarity, Dover Publications, Mineola, NY, USA, 2002.

[20] B. A. Pethica, "Contact angles," in Reports on the Progress of Applied Chemistry, vol. 46, pp. 14-17, Society of Chemical Industry, London, UK, 1961.

[21] B. M. Law, S. P. McBride, J. Y. Wang et al., "Line tension and its influence on droplets and particles at surfaces," Progress in Surface Science, vol. 92, no. 1, pp. 1-39, 2017.

[22] V. A. Nebol'sin, A. A. Shchetinin, and E. I. Natarova, "Variation in silicon whisker radius during unsteady-state growth," Inorganic Materials, vol. 34, no. 2, pp. 87-89, 1998.

[23] V. V. Voronkov, "On the thermodynamic equilibrium at a linear junction of three phases," Fiz. Tverd. Tela, vol. 5, no. 2, pp. 571-574, 1963.

[24] V. A. Nebol'sin, A. A. Shchetinin, T. I. Sushko, and P. Yu. Boldyrev, "Constant growth angle for silicon whiskers," Inorganic Materials, vol. 32, no. 9, pp. 1031-1034, 1996.

[25] F. Panciera, Z. Baraissov, G. Patriarche et al., "Phase selection in self-catalyzed GaAs naniwires//," Nano Lett, 2020.

[26] V. A. Nebol'sin, D. B. Suyatin, E. V. Zotova, and S. S. Shmakova, "Metal solvent droplet stability in silicon whisker growth," Inorganic Materials, vol. 48, no. 9, pp. 861-866, 2012.

[27] F. M. Ross, J. Tersoff, and M. C. Reuter, "Sawtooth faceting in silicon nanowires," Physical Review Letters, vol. 95, no. 14, p. 146104, 2005.

[28] W. K. Burton, N. Cabrera, and F. C. Frank, "The growth of crystals and the equilibrium of their surfaces," Philosophical Transactions of the Royal Society A, vol. 243, pp. 299-358, 1951.

[29] V. G. Dubrovskii, "Development of growth theory for vaporliquid-solid nanowires: contact angle, truncated facets, and crystal phase," Crystal Growth \& Design, vol. 17, no. 5, pp. 2544-2548, 2017.

[30] V. G. Dubrovskii, N. V. Sibirev, N. N. Halder, and D. Ritter, "Classification of the morphologies and related crystal phases of III-V nanowires based on the surface energy analysis," The
Journal of Physical Chemistry C, vol. 123, no. 30, pp. 1869318701, 2019.

[31] V. A. Nebol'sin, A. I. Dunaev, A. S. Samofalova, and V. V. Korneeva, "Contact interaction in an $\mathrm{M}-\mathrm{SiO}_{2}(\mathrm{M}=$ metal catalyst for nanowhisker growth) system," Inorganic Materials, vol. 54, no. 6, pp. 558-563, 2018.

[32] E. I. Givargizov, "Periodic instability in whisker growth," Journal of Crystal Growth, vol. 20, no. 3, pp. 217-226, 1973.

[33] E. I. Givargizov and N. N. Sheftal', "Morphology of silicon whiskers grown by the VLS-technique," Journal of Crystal Growth, vol. 9, pp. 326-329, 1971.

[34] S. Q. Feng, D. P. Yu, H. Z. Zhang, Z. G. Bai, and Y. Ding, "The growth mechanism of silicon nanowires and their quantum confinement effect," Journal of Crystal Growth, vol. 209, no. 23, pp. 513-517, 2000. 